\title{
UVA-1-Phototherapie in der Dermatologie: 10 Jahre klinische Erfahrungen
}

\section{Kowalzick}

\author{
UVA-1-Phototherapy in Dermatology: 10 Years of Experience
}

\section{Zusammenfassung}

Die breitesten klinischen Erfahrungen sowie mehrere kontrollierte Studien liegen zur UVA-1-Phototherapie des atopischen Ekzems vor; diese Indikation gilt, zumindest für schwere, akut exazerbierte Fälle, als gesichert. Als weitere Indikationen ergeben sich aufgrund von Fallstudien das dyshidrotische Handekzem, die zirkumskripte und systemische Sklerodermie, der subakut kutane und systemische Lupus erythematodes, das kutane T-Zell-Lymphom, die Urticaria pigmentosa und die Psoriasis. Als mögliche weitere Indikationen der UVA-1-Phototherapie wurden aufgrund positiver Fallbeobachtungen die eosinophile Follikulitis, das Hypereosinophilie-Syndrom, die retikuläre erythematöse Muzinose, die Pityriasis lichenoides chronica, die Pityriasis lichenoides et varioliformis acuta, die kleinherdige Parapsoriasis Brocq, die Mucinosis follicularis, der Lichen sclerosus et atrophicus, die Graft-versus-Host-Disease, das POEMS-Syndrom sowie Keloide vorgeschlagen.

\section{Abstract}

Most clinical experiences and several controlled studies exist concerning UVA-1 phototherapy of atopic dermatitis. This indication is generally accepted at least in severe acute cases. Further encouraging results with UVA-1 phototherapy were obtained in clinical case studies on dyshidrotic hand eczema, circumscribed and systenic scleroderma, sbubacute cutaneous and systemic lupus erythematosous, and cutaneous T-cell lymphoma, urticaria pigmentosa and psoriasis. Other possible future indications for UVA-1 phototherapy based on case reports are eosinophilic folliculitis, hypereosinophilia, reticular erythematsous mucinosis, pityriasis lichenoides chronica, pityriasis lichenoides et varioliformis acuta, small plaque type parapsoriasis Brocq, mucinosis follicularis, lichen sclerosus et atrophicus, graft versus host disease, POEMS syndrome and keloids.

\section{Einleitung}

Bereits in den 30er- und 40er-Jahren des letzten Jahrhunderts wurden Patienten mit atopischem Ekzem mittels Kohlenbogenlampen behandelt, die retrospektiv als UVA-1-Geräte klassifiziert werden können. 1981 beschrieben Mutzhas et al. eine Halogenhochdruck-Strahler-Apparatur, die vorzugsweise UVA-1 emittierte und zunächst besonders zur Diagnostik von Photodermatosen eingesetzt wurde [34]. Vor 10 Jahren wurden dann die ersten kontrollierten Studien zur Phototherapie von Hautkrank- heiten mit UVA-1 publiziert. Die UVA-Strahlung wird in das kürzerwellige UVA-2 (320 nm bis $340 \mathrm{~nm}$ ) und das langwellige UVA-1 (340 nm bis $400 \mathrm{~nm}$ ) eingeteilt. Grund dafür ist u.a., dass die Aktionsspektren verschiedener unerwünschter UV-bedingter Effekte (Erythem, Photokarzinogenese, Hautalterung) nach ihrem Maximum im UV-B-Bereich im UV-A2-Bereich noch steil abfallen und bei ca. $340 \mathrm{~nm}$ ein Plateau auf niedrigem Niveau (etwa $10^{-3}$ bis $10^{-4}$ der maximalen Dosiswirkung) erreichen (Abb.1). Wegen der vergleichsweise geringen phototoxischen Wirksamkeit der UVA-1-Strahlung können in kurzer Zeit sehr

Klinik für Hautkrankheiten und Allergologie, Vogtland-Klinikum Plauen GmbH

(Chefarzt: Prof. Dr. med. L. Kowalzick)

Korrespondenzadiresse

Prof. Dr. med. habil. L. Kowalzick · Klinik für Hautkrankheiten und Allergologie, · Vogtlandklinikum Plauen

GmbH· Maximilian-Kolbe-Weg 1a·08529 Plauen·E-mail: 1.kowalzick@vogtland-klinikum-plauen.de

Bibliografie

Akt Dermatol 2004; 30: 62-68 @ Georg Thieme Verlag Stuttgart · New York · ISSN 0340-2541 .

DOI 10.1055/s-2004-814229 


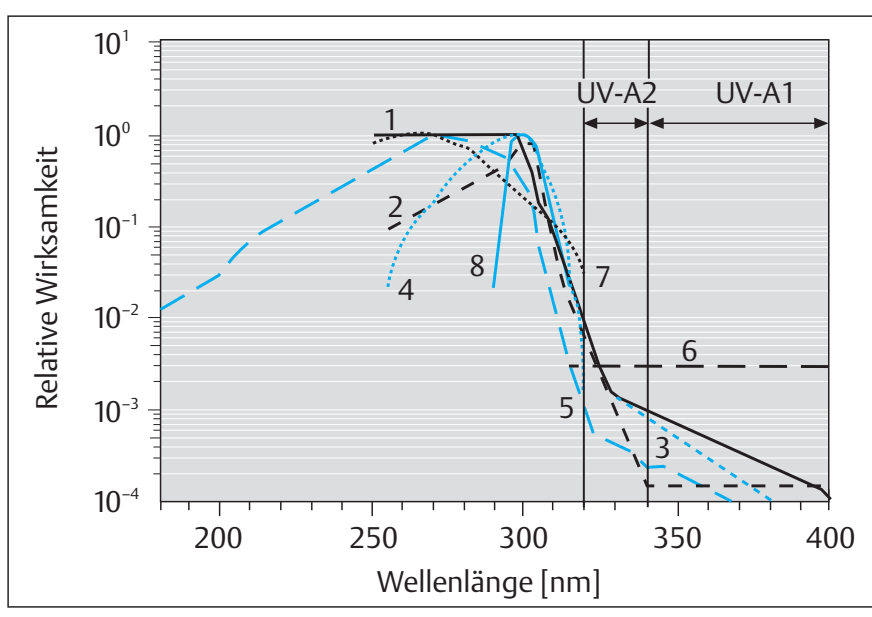

Abb. 1 Aktionsspektren (relative spektrale Wirksamkeiten) verschiedener erwünschter und unerwünschter Effekte von ultravioletter Strahlung nach CIE (Konföderation internationaler Ingenieure): Erythembildung (1) menschlicher Haut; Photokarzinogenese (2) und photoinduzierte Hautalterung (3) haarloser Albinomäuse; Vitamin-D3-Synthese (4) menschlicher Haut; Gesundheitsgefährdung durch Hautbestrahlung (5), Augenbestrahlung (6) und Immunsuppression (7); antipsoriatrische Wirksamkeit (8). Zu beachten ist die zehnerlogarithmische Auftragung der y-Achse. Modifiziert nach Meffert et al. [31].

hohe Dosen therapeutisch appliziert, und spezifische photobiologische Effekte erreicht werden. In der klinischen Anwendung werden UVA-1-Bestrahlungen nach verschiedenen Dosierungsschemata eingesetzt, die teilweise auch durch die Leistungsfähigkeit der zur Verfügung stehenden Strahler (Fluoreszenzbzw. Halogenhochdruck-Strahler) begrenzt werden. Es wird üblicherweise differenziert zwischen Niedrigdosis- (10 bis $20 \mathrm{~J} / \mathrm{cm}^{2}$ pro Einzelbestrahlung) Mitteldosis- (30 bis $70 \mathrm{~J} / \mathrm{cm}^{2}$ ) und Hochdosis- $\left(130 \mathrm{~J} / \mathrm{cm}^{2}\right)$ UVA-1-Therapie. Im Folgenden werden die Möglichkeiten zur UVA-1-Phototherapie von Hautkrankheiten sowie ihre Risiken und Nebenwirkungen bzw. Kontraindikationen dargelegt und Hinweise zu deren Wirkmechanismen gegeben.

\section{Atopisches Ekzem}

\section{Hochdosis-Therapie}

1992 berichteten Krutmann und Mitarbeiter über eine randomisierte Untersuchung [26], in der eine kombinierte UVA- und UVB-Bestrahlung mit einer Hochdosis-UVA-1-Behandlung bei Patienten mit schwerem, akut exazerbierten, atopischen Ekzem über jeweils 15 Tage verglichen wurde. Die UVA-1-Behandlung erhielten 15 Patienten mit $15 \times 130 \mathrm{~J} / \mathrm{cm}^{2}$. Eine sonstige spezifische Behandlung des Ekzems erfolgte nicht. Unter diesen Studienbedingungen erwies sich die UVA-1-Therapie unter Verwendung eines klinischen Schwere-Scores nach da Costa als signifikant überlegen. In der Folge wurde, an diese monozentrische Pilotstudie anschließend, eine randomisierte Multizenterstudie [27] mit Hochdosis-UVA-1 (130 J/ $\mathrm{cm}^{2}, 20$ Patienten) durchgeführt. Als Kontrollgruppen dienten wiederum Patienten mit kombinierter UVA/B -Therapie sowie eine Gruppe mit topischer Fluocortolon-Behandlung. Nach jeweils 10-tägiger Behandlung waren unter Zugrundelegung des klinischen Score-Systems die
UVA-1- und die Steroidtherapie hochsignifikant der kombinierten UVA/B-Bestrahlung überlegen. Beim Vergleich der UVA-1Therapie mit der Steroidbehandlung fand sich erstere wiederum signifikant überlegen. Eine signifikante Reduktion des ECP im Serum fand sich nur bei diesen beiden Therapieverfahren. Kürzlich wurde eine Studie publiziert [5], in der 11 Patienten mit UVA1-Einzeldosen von $130 \mathrm{~J} / \mathrm{cm}^{2}$ über dreizehn Tagen behandelt wurden. An den ersten beiden Behandlungstagen erhielten die Patienten 60 bzw. $90 \mathrm{~J} / \mathrm{cm}^{2}$. Hierbei nahm der SCORAD-Schwereindex hochsgnifikant ab. Ein signifikanter Einfluss auf den ECP Spiegel im Serum ließ sich jedoch nicht nachweisen.

\section{Mitteldosis- Therapie}

Im gleichen Jahr wie die erste Hochdosis-Studie wurden auch die Ergebnisse einer ersten Untersuchung [30] mit UVA-1 im mittleren Dosisbereich publiziert. Hier wurde die Dosis anfänglich täglich gesteigert, im Schnitt erhielten die insgesamt 30 über 10 Tage behandelten Patienten jeweils $36 \mathrm{~J} / \mathrm{cm}^{2}$. Bei diesen Patienten nahm der klinische Schwere-Score nach da Costa gleichfalls hochsignifikant ab. Eine weitere Untersuchergruppe [3] behandelte 19 Patienten ebenfalls mit initialer Dosissteigerung mit durchschnittlich $38 \mathrm{~J} / \mathrm{cm}^{2}$ über 15 Tage. Auch hier fanden sich die Patienten nach diesem klinischen Score-System hochsignifikant gebessert. Bei 22 weiteren Patienten mit akut exazerbierten atopischen Ekzemen wurde eine offene Studie mit 16-maliger Applikation von tgl. 27,6 J/ $\mathrm{cm}^{2}$ bei unspezifischer Begleitmedikation durchgeführt. Hierunter nahm der durchschnittliche SCORAD-Wert hochsignifikant ab. Ein Einfluss auf ECP und sIL-2-Rezeptor fand sich nicht [6].

In eigenen Untersuchungen $[15,16]$ wurden UVA-1-Dosierungen von $35 \mathrm{bzw} .50 \mathrm{~J} / \mathrm{cm}^{2}$ pro Bestrahlung angewandt. Alle (11 bzw. 13) Patienten erhielten 15 Bestrahlungen ohne Dosissteigerung. Eine spezifische Begleittherapie der Patienten mit schweren, akut exazerbierten atopischen Ekzem erfolgte nicht. In beiden Dosierungsgruppen ließ sich eine signifikante Besserung des klinischen Schweregrades nach dem SCORAD-Index feststellen. Die klinische Befundbesserung korrelierte signifikant mit der Abnahme des löslichen Interleukin-2-Rezeptors im Serum der Patienten, einem Marker der Krankheitsaktivität, dagegen nicht mit einer Abnahme des ECP-Spiegels. In einer weiteren Studie [11], bei der Einzeldosen von $50 \mathrm{~J} / \mathrm{cm}^{2}$ gleichfalls über 15 Tage gegeben wurden, nahm bei jeweils 50 mit zwei verschiedenen UVA-1-Quellen behandelten Patienten der durchschnittliche SCORAD-Index jeweils signifikant ab. Eine Kontrollgruppe, die mit einer kombinierten UVA/B-Bestrahlung behandelt wurde, war den UVA-1-therapierten Kollektiven jeweils signifikant unterlegen. In den beiden UVA-1-Gruppen nahm der Serum-Spiegel des sIL-2-Rezeptors und des sIL-4-Rezeptors jeweils signifikant ab. Mit der Dosis von $50 \mathrm{~J} / \mathrm{cm}^{2}$ wurden in einer weiteren Untersuchung [2] 32 Patienten jeweils 15-mal bestrahlt. Der SCORAD-Wert nahm signifikant ab. In einer weiteren Studie wurden 12 Patienten mit UVA-1 in Einzeldosen von jeweils $65 \mathrm{~J} / \mathrm{cm}^{2}$ über 15 Tage therapiert. Hierunter nahm der SCORAD-Index gleichfalls signifikant ab [5]. Zusammenfassend lässt sich festhalten, dass betreffend die Mitteldosis-UVA-1-Therapie des atopischen Ekzems die umfangreichsten Ergebnisse publiziert wurden, und hierbei speziell das von uns entwickelte Schema von $15 \times$ $50 \mathrm{~J} / \mathrm{cm}^{2}$ Verwendung fand. 


\section{Niedrigdosis-Therapie}

Der Effekt der niedrig dosierten UVA-1-Therapie beim atopischen Ekzem wurde nur im Rahmen von Dosisvergleichsstudien untersucht. Es wurden Einzeldosen von 10 [15] bzw. $20 \mathrm{~J} / \mathrm{cm}^{2}$ [5] jeweils 15-mal bei 13 bzw. 11 Patienten mit schwerem akut exazerbierten atopischen Ekzem gegeben. Der SCORAD-Index nahm in beiden Untersuchungen unter der Therapie von 64 auf 51 bzw. von 55 auf 47 Punkten ab, wobei eine statistische Signifkanz der Abnahme nur in der ersten Gruppe zu verzeichnen war.

\section{Dosisvergleiche}

Die UVA-1-Therapie ist in der Therapie des akut exazerbierten atopischen Ekzems in allen drei untersuchten Dosisbereichen bei 15-maliger Anwendung nachweisbar wirksam. Zumindest die mittel und hoch dosierte UVA-1-Therapie sind einer kombinierten UVA/B-Therapie signifikant überlegen. Wegen möglicher Risiken und Nebenwirkungen auch einer UVA-1-Therapie ist es wünschenwert, die niedrigste optimal wirksame Einzeldosis zu ermitteln. In Dosisvergleichsstudien wurde festgestellt, dass $10 \mathrm{~J} / \mathrm{cm}^{2}$ signifikant weniger wirksam sind als $50 \mathrm{~J} / \mathrm{cm}^{2}$ [15] und dass im Bereich von $10 \mathrm{~J} / \mathrm{cm}^{2}$ über $35 \mathrm{~J} / \mathrm{cm}^{2}$ bis $50 \mathrm{~J} / \mathrm{cm}^{2}$ die Dosis signifikant mit der klinischen Wirksamkeit korreliert [19]. In einer weiteren Untersuchung wurden Einzeldosen von $65 \mathrm{~J} / \mathrm{cm}^{2}$ mit $130 \mathrm{~J} / \mathrm{cm}^{2}$ verglichen, wobei die höhere Dosierung zwar etwas stärker wirksam, ein statistisch signifikanter Unterschied zwischen beiden Gruppen aber nicht errechnet wurde [5]. Mit den gleichen Dosen wurden Patienten in einer Halbseitenvergleichsstudie behandelt [47]. Hierbei reduzierte der SCORADWert in der Hochdosis-Gruppe im Mittel um 35,5\%, in der Mitteldosisgruppe um 31,4\%, der Unterschied war statistisch nicht signifikant. Diese Daten legen nahe, dass die Mitteldosis-Therapie mit 15 Bestrahlungen à $50-65 \mathrm{~J} / \mathrm{cm}^{2}$ das optimale Behandlungsschema für eine UVA-1-Therapie des atopischen Ekzems darstellt.

\section{Dauer des Therapieeffekts}

Über die Dauer des Therapieeffektes nach UVA-1-Therapie liegen mehrere Untersuchungen vor. In drei wurden jeweils $50 \mathrm{~J} / \mathrm{cm}^{2}$ als Einzeldosis 15 -mal appliziert $[2,13,18]$. Bei den jeweils 32,10 bzw. 100 nachbeobachteten, lediglich unspezifisch hautpflegend nachbehandelten Patienten wurde 4 bzw. 6 Wochen nach Therapieende noch eine signifikante Befundbesserung verglichen mit dem Ausgangsbefund gesehen. Diese fand sich nach 12 Wochen in einer der Untersuchungen [2] jedoch nicht mehr. In einer anderen Studie zeigten sowohl Hochdosis als auch Mitteldosis einen über gleichfalls mindestens 4 Wochen anhaltenden Effekt [47]. In jedem Falle ist ein Wiederaufflammen der Symptomatik kurz nach Therapieende, anders als beispielweise nach kombinierter UVA/B-Therapie [13], nicht zu beobachten. Zum Teil weisen die Patienten nach UVA-1-Therapie Rezidivfreiheit von $1 \mathrm{Jahr}$ Dauer und mehr auf.

\section{Responder und Non-Responder}

Das therapeutische Ansprechen auf die UVA-1-Therapie ist nicht bei allen Patienten gleich gut. Neben den sog. Respondern gibt es einen Anteil von Non-Respondern, die nur geringe Besserung, einen gleich bleibenden Befund oder eine (geringfügige) Verschlechterung unter der Therapie erfahren. Dieser Anteil beträgt bei der Mitteldosis-Therapie zwischen 19 und 30\% [3,21,30]. Genaue Angaben über den Prozentsatz von Non-Repondern bei der
Hochdosis-Therapie im Rahmen von Studien fehlen bislang, jedoch wurde in einer offenen Untersuchung $25 \%$ Non-Responder angegeben [40]. In einer ausführlichen Charakterisierung dieser Non-Responder wurde gefunden, dass diese einen signifikant höheren klinisch-anamnestischen Erlanger Atopiekriterien-Score-Wert, ein signifikant höheres Serum Gesamt-IgE, eine signifikant höhere Zahl hochtitriger, spezifischer IgE-Spezies gegenüber sog. Atopenen und eine häufigere Besiedlung der Haut mit Staphylococcus aureus bzw. des Darmes mit Candida albicans, jeweils verglichen mit Respondern, hatten [40]. In einer eigenen Untersuchung konnten wir keinen Anhalt dafür finden, dass klinisch schwerere Fälle von atopischen Ekzem oder solche mit hohem Gesamt-IgE schlechter auf eine Mitteldosis-Therapie ansprechen würden als leichtere Fälle [17]. Interessant wäre eine Analyse, ob Patienten mit bevorzugtem Befall frei getragener Hautpartien, wie beispielsweise des Gesichtes und Halses (headand-neck-type), schlechter auf eine UVA-1-Therapie ansprechen als andere Lokalisationsmuster der Erkrankung. Hiergegen sprechen zumindest Beobachtungen von Göring [6].

\section{Wirkungsmechanismen}

Die meisten Untersuchungen zu den möglichen Wirkungsmechanismen beim atopischen Ekzem wurden mit HochdosisUVA-1 in vivo und in vitro durchgeführt. Möglicherweise gibt es hier Unterschiede zum niedriger dosierten Bereich: Während z. B. Hochdosis-UVA-1 in vivo die Aktivität von Langerhanszellen beeinträchtigt, fanden sich solche Effekte in vitro im MitteldosisBereich-Therapie nicht. Hochdosis-UVA-1 senkt die Zahl von IgE-Rezeptoren tragender Langerhanszellen und Mastzellen in Epidermis und unterdrückt vorher positive so genannte epikutane Atopie-Patch-Reaktionen gegen inhalative Antigene bei entsprechend sensibilisierten Atopie-Patienten. Hochdosis-UVA-1 reduziert die Expression des Th1-typischen Zytokins Gamma-Interferon sowie des Adhäsionsmoleküls ICAM-1 in läsionaler Haut von Patienten mit atopischem Ekzem und lässt die Expression von Th2-typischen Zytokinen wie Interleukin-4 unberührt. Dagegen wird in vitro die Expression des immunsuppressiv wirksamen Zytokins Interleukin-10 durch UVA-1 erhöht. Reguliert werden diese Änderungen der Expression wahrscheinlich auf GenEbene durch die Aktivierung des Transkriptionsfaktors AP2 durch von UVA-1 induzierten oxidativen Mechanismen (Singulett-Sauerstoff) in der Haut. Darüber hinaus führen wahrscheinlich diese oxidativen Mechanismen zur Apoptose (programmierten Zelltod) von T-Helfer-Lymphozyten in läsionaler Haut von Patienten mit atopischem Ekzem.

\section{Dyshidrotisches Elkzem}

In einer Pilotuntersuchung [41] wurden 12 Patienten mit akut exazerbiertem chronischem dyshidrotischen Handekzem mit UVA-1 behandelt. Steroide oder Antihistaminika wurden nicht gegeben. Es erfolgten 15 Bestrahlungen mit jeweils $40 \mathrm{~J} / \mathrm{cm}^{2}$ über drei Wochen. Bei 10 der beahandelten Patienten trat eine weitgehende Abheilung der Hautsymptome einschließlich des Juckreizes ein. Unter Verwendung eines Score-Systems (DASI) war der Therapieerfolg signifikant nachweisbar. Der Behandlungserfolg hielt bei diesen Respondern über eine Zeit von drei Monaten nach Behandlungsende an. 
5-mal wöchentlich über 3 Wochen. Unter Verwendung eines klinischen Score-Systems besserten sich unter der Therapie 9 von

In einer Pilotstudie [45] wurden 10 Patienten mit zumeist multilokulärer/generalisierter zirkumskripter Sklerodermie (Morphea) lokal einer Hochdosis-UVA-1-Therapie mit insgesamt $30-$ mal $130 \mathrm{~J} / \mathrm{cm}^{2}$ unterzogen. Alle behandelten Plaques sprachen an, eine vollständige Rückbildung erfolgte bei 4 Patienten. Nicht bestrahlte Herde blieben unverändert. Die Befundbesserungen waren auch in der 20-MHz-Sonographie der Haut objektivierbar. Eine Behandlung bei 7 weiteren Patienten mit einer niedrigen Dosierung von $20 \mathrm{~J} / \mathrm{cm}^{2}$ zeigte nur 2 partielle Responder. Dagegen fand eine andere Untersuchergruppe die Bestrahlungsbehandlung mit niedrig dosiertem UVA-1 $\left(20 \mathrm{~J} / \mathrm{cm}^{2}\right)$ ebenfalls 30-mal, über einen Zeitraum von 12 Wochen verteilt, gegeben, bei allen 20 therapierten Patienten als wirksam, mit einer weitgehenden, objektivierbaren Besserung, jedoch ohne vollständige Abheilung aller Herde. Bei den weitaus meisten Patienten war der Therapieerfolg über zumindestens 3 Monate nach Therapieende hinaus stabil [10]. Bei 4 von 7 in einer Untersuchung mit $30 \times 30 \mathrm{~J} / \mathrm{cm}^{2}$ behandelten Patienten bildeten sich die Morpheaherde vollständig, bei den 3 anderen weitgehend zurück [4]. In einer Studie an 19 Kindern zwischen 3 und 13 Jahren mit Morphea wurde mit niedrig dosiertem UVA-1 in einer Dosis von $20 \mathrm{~J} / \mathrm{cm}^{2}$ viermal wöchentlich über 10 Wochen behandelt. Zusätzlich wurden sie topisch zweimal täglich mit CalcipotriolSalbe behandelt. Nach dieser Behandlungsphase zeigte sich eine signifikante Besserung eines klinischen Scores [23]. In einer Einzelfallbeschreibung berichten die Autoren auch über den erfolgreichen Einsatz dieses Schemas bei einem besonders schweren Fall von pansklerotischer Morphea bei einem Kind [46]. Wir behandelten einen Fall von Sklerodermie en coup de sabre bei einer Heranwachsenden ebenfalls mit gutem Resultat mit einer Kombination von topischem Calcipotriol und $30-\mathrm{mal} 30 \mathrm{~J} / \mathrm{cm}^{2}[22]$.

Bei 4 Patienten mit progressiver systemischer Sklerodermie (PSS) wurde täglich eine Teilkörper-Bestrahlung im Bereich der Unterarme und Hände mit $60 \mathrm{~J} / \mathrm{cm}^{2}$ UVA-1 durchgeführt. Nach zwischen 9 und 29 Sitzungen zeigten sich die Thermographie und die Haut-Elastizität wie die Fingerstreckbarkeit jeweils signifikant verbessert [33]. Entsprechend berichtet von Kobyletzki [14] über die erfolgreiche lokale Behandlung mit signifikanter Abnahme eines klinischen Scores bei 8 Patienten mit Akrosklerose bei PSS mittels niedrig dosiertem UVA-1 $\left(30 \mathrm{~J} / \mathrm{cm}^{2}\right.$ über 50 Sitzungen).

Als Wirkungsmechanismus wird die nachgewiesene Induzierbarkeit von Kollagenase I in dermalen Fibroblasten durch UVA-1, sowohl in vivo wie in vitro nachweisbar, vermutet. Unter UVA-1 nehmen Kollagen I und III in der Haut ab. Ein möglicherweise zusätzlich bestehender immunsuppressiver Einfluss auf das entzündliche Infiltrat sowie auf die Zahl der CD34+ dendritischen Zellen bei Morphea ist ebenfalls denkbar.

\section{Lupus erythematodes}

Eine amerikanische Arbeitsgruppe berichtete 1992 erstmals über die Therapie des systemischen (SLE) bzw. subakut kutanen Lupus erythematodes (SCLE) mit niedrig dosiertem UVA-1 bei 10 Patienten. Diese erhielten durchschnittliche Dosen von $6 \mathrm{~J} / \mathrm{cm}^{2}$,
10 Patienten um durchschnittlich 39\%, die 5 Patienten mit Hautbeteiligung besserten sich, allerding nicht signifikant, um $25 \%$. Vier SSA-Antikörper-positive Patienten wurden negativ; bei zwei Patienten sank der ANA-Titer [28]. In einer weiteren Kasuistik [42] wurde über das gute Ansprechen einer Patientin mit SCLE auf eine Niedrigdosis UVA-1-Therapie berichtet. Bestrahlt wurde über einen Zeitraum von 9 Wochen mit zunächst $21 \mathrm{x}$ $2,6 \mathrm{~J} / \mathrm{cm}^{2}$, anschließend mit $11 \times 11,9 \mathrm{~J} / \mathrm{cm}^{2}$. Binnen 14 Wochen kam es zu einer völligen Rückbildung der Hautveränderungen, die über mehr als 12 Monate anhielt. Die Titer antinukleärer Antikörper blieben gleichwohl unverändert (1:400) positiv. In einer plazebokontrollierten Studie mit crossover-Design [29] wurden 26 Frauen mit SLE jeweils für 3 Wochen 5 -mal wöchentlich mit $6 \mathrm{~J} / \mathrm{cm}^{2}$ UVA-1 oder sichtbarem Licht (Wellenlänge $>430 \mathrm{~nm}$ ) bestrahlt. Anschließend wurden die Patientinnen 3 Wochen mit der jeweils anderen Modalität bestrahlt. Nur in der jeweils mit UVA-1 behandelten Gruppe kam es zu einer signifikanten Besserung gemessen an einem Score-System (SLAM). Der Verbrauch an Antirheumatika nahm ab und die dsDNS-Autoantikörper-Titer sanken signifikant. Binnen drei Wochen nach Ende der UVA1-Therapie kam es zu einer Wiederverschlechterung der Symptomatik. Eine erneute Therapie mit 3 Bestrahlungen UVA-1 wöchentlich führte zu einer erneuten Besserung binnen 6 Wochen. Eine Behandlung mit jeweils 6 bis $15 \mathrm{~J} / \mathrm{cm}^{2}$ UVA-1 ein- bzw. zweimal wöchentlich war als Erhaltungstherapie über durchschnittlich mindestens 3,4 Jahre wirksam [32]. In einer weiteren Studie wurden 11 Patienten mit SLE nach einem ähnlichen Studiendesign mit niedrig dosiertem UVA-1 behandelt. Im Gegensatz zu den Behandlungsphasen mit sichtbarem Licht kam es während der jeweils dreiwöchigen UVA-1-Therapie zu einem signifikanten Rückgang der SLAM- bzw. SLEDAI- Score-Werte [38].

\section{Mycosis fungoides}

In einer offenen Pilotstudie wurden 3 Patienten mit kutanem T-Zell-Lymphom (CTCL) vom Typ der Mycosis fungoides (MF) in den Stadien Ia und Ib mit täglich jeweils $130 \mathrm{~J} / \mathrm{cm}^{2} \mathrm{bzw} .60 \mathrm{~J} / \mathrm{cm}^{2}$ UVA-1 für 20 bzw. 16 Sitzungen behandelt. Unter dieser Therapie heilten die kutanen Lymphomherde histologisch verifiziert jeweils komplett ab (komplette Remission) [36]. In einer Einzelfallbeobachtung wurde gleichfalls 1999 das gute Ansprechen eines Patienten im Stadium III auf $15 \times 60 \mathrm{~J} / \mathrm{cm}^{2}$ berichtet [11]. In einer weiteren Studie wurden 13 Patienten mit MF in den Stadien Ib bis III täglich mit $100 \mathrm{~J} / \mathrm{cm}^{2}$ UVA-1 für 10 bis 35 (im Mittel 22) Sitzungen bestrahlt. 11 der Patienten zeigten unter der Therapie eine histologisch gesicherte komplette Remission, zwei (je ein Patient im Stadium Ib und IIb) zeigten eine partielle Remission ihrer kutanen Herde [49].

In einem anderen Fall zeigte ein Patient nach 67 Behandlungen von zuletzt 5-mal wöchentlich $80 \mathrm{~J} / \mathrm{cm}^{2}$ zwar klinisch eine komplette Remission seiner Stadium-la-Erkrankung, war histologisch jedoch noch keineswegs tumorfrei, weswegen genaue bioptische Kontrollen eines klinischen Behandlungserfolges dringend empfohlen werden [43]. Darüber hinaus wurde über das fast vollständige Ansprechen eines Falls von kleinherdiger Parapsoriasis Brocq, einem möglichen Vorläuferstadium der MF, 
auf eine Therapie mit $15-\mathrm{mal} 50 \mathrm{~J} / \mathrm{cm}^{2}$ [24] sowie über das klinisch vollständige Ansprechen einer Mucinosis follicularis, einer gleichfalls häufig mit der MF assoziierten Erkrankung, auf eine Therapie mit $15-\mathrm{mal} 60 \mathrm{~J} / \mathrm{cm}^{2}$ berichtet [12]. Als Wirkmechanismus der UVA-1-Therapie beim kutanen T-Zell-Lymphom wird die Induktion der Desoxyribonuklease-abhängigen Apoptose der Tumorzellen vom T-Helfer-Zell-Phänotyp durch UVA-1 angenommen. Diese konnte in vivo unter einer Dosis von $60 \mathrm{~J} / \mathrm{cm}^{2}$ UVA-1 nachgewiesen werden.

\section{Urticaria pigmentosa}

In einer Pilotuntersuchung [44] wurden Patienten mit Urticaria pigmentosa (kutaner Mastozytose) mit UVA-1 therapiert. Nach initialer einmaliger Bestrahlung mit $60 \mathrm{~J} / \mathrm{cm}^{2}$ wurden weitere 9 Bestrahlungen mit je $130 \mathrm{~J} / \mathrm{cm}^{2}$ Hochdosis-UVA-1 gegeben. Bereits nach 4 Bestrahlungen war das Darier-Zeichen nicht mehr auslösbar. Bemerkenswerterweise schien der Besserungseffekt, im Gegensatz zur PUVA-Therapie länger, zumindest über 2 Jahre nach Absetzen der UVA-1-Therapie fortzubestehen. Als Wirkmechanismus wird die zahlenmäßige Reduktion dermaler Mastzellen unter Hochdosis-UVA-1 in vivo vermutet. Diese guten, lang anhaltenden therapeutischen Ergebnisse konnten von anderen Autoren $[20,48]$ bei Einsatz der Hochdosis- bzw. MitteldosisUVA-1-Therapie nicht nachvollzogen werden.

\section{Psoriasis}

In einer kontrollierten Halbseiten-Studie [19] an drei Patienten wurde die Wirkung von UVB- (Breitspektrum) und MitteldosisUVA-1-Bestrahlungen bei drei Patienten mit schwerer Plaque Psoriasis verglichen. Die Patienten erhielten auf der UVA-1 behandelten Körperseite täglich Bestrahlungen mit $50 \mathrm{~J} / \mathrm{cm}^{2}$ über 15 Tage. An beiden Körperseiten kam es zu einer signifikanten Abhahme des PASI-Scores, die UVB behandelte Seite war jedoch, wenn auch nicht signifikant, stärker gebessert. Die prinzipielle Wirksamkeit der Mitteldosis-UVA-1-Therapie ist jedoch beispielweise für Patienten mit HIV-Infektion und gleichzeitig bestehender Psoriasis von Interesse, da UVA-1, anders als UV-B kombiniert mit UVA-2, das HIV-Promotor-Gen nicht aktiviert. So konnte in ersten Pilotuntersuchungen gezeigt werden, dass UVA-1-Bestrahlungen von HIV-Patienten mit Psoriasis wirksam waren und keine Erhöhung der HIV-Genom-Kopien im Blut zur Folge hatten (persönliche Mitteilung, J. Krutmann, EADV-Kongress, Genf 2000).

\section{Sonstige Indikationen}

Über die erfolgreiche Therapie eines Falles von retikulärer erythematöser Muzinose (REM-Syndrom) nach 15 Sitzungen mit je $50 \mathrm{~J} / \mathrm{cm}^{2}$ UVA-1 wurde berichtet [8]. Bei drei Patienten mit Hypereosinophilie-Syndrom wurde nach 15 Bestrahlungen mit je $50 \mathrm{~J} / \mathrm{cm}^{2}$ UVA-1 ein Rückgang der Hautveränderungen, aber auch ein deutlicher Rückgang der Blut-Eosinophilen, der HautEosinophilen und des ECP im Serum erreicht, die 2 bis 18 Monate anhielten [37]. Drei Patienten mit Pityriasis lichenoides et varioliformis acuta (PLEVA) und drei von fünf Patienten mit Pityriasis lichenoides chronica heilten unter einer Mitteldosis-Therapie mit Einzeldosen von $60 \mathrm{~J} / \mathrm{cm}^{2}$ UVA-1 vollständig ab [35]. Über den erfolgreichen Einsatz von Einzeldosen von $20 \mathrm{~J} / \mathrm{cm}^{2}$ UVA-1 bei Lichen sclerosus et atrophicus wurde berichtet [25]. In einem Fall von sklerodermiformer Graft-versus-host-Reaktion konnte mit 24 Bestrahlungen mit $20 \mathrm{~J} / \mathrm{cm}^{2}$ UVA- 1 bei gleichzeitiger Therapie mit Mycophenolat Mofetil nach 6 Wochen eine deutliche Verbesserung der Hautveränderungen erzielt werden [7]. Bei einem Patienten mit POEMS-Syndrom konnten die sklerodermiformen Hautveränderungen mit 35 Bestrahlungen mit $30 \mathrm{~J} / \mathrm{cm}^{2}$ UVA-1 gebessert werden [39]. In einem Fall eines Keloids wird über die deutliche Abflachung nach 22 Bestrahlungen von jeweils $130 \mathrm{~J} / \mathrm{cm}^{2}$ UVA-1 berichtet [1], bei drei weiteren Patienten konnte jedoch mit 15 bis $18-\mathrm{mal} 100 \mathrm{~J} / \mathrm{cm}^{2}$ keine objektive Befundbesserung erzielt werden [9].

\section{Nebenwirkungen, Risiken und Kontraindilkationen}

Die akuten Nebenwirkungen einer UVA-1-Therapie sind limitiert. Die Augen sind stets durch UV-A-undurchlässige Schutzbrillen, wie sie auch in Solarien verwendet werden, zu schützen. Die meisten Patienten mit atopischem Ekzem vertragen initial Dosen von über $90 \mathrm{~J} / \mathrm{cm}^{2}$ UVA-1 ohne jede Erythemwirkung [15]. Gleichwohl ist die Bestrahlung eines Probefeldes bzw. die Bestimmung der minimalen erythemwirksamen/phototoxischen Dosis (MED/MPD) vor Ganzkörper-Therapiebeginn mit Mittelbzw. Hochdosis-UVA-1 dringend zu empfehlen. Eine meist recht starke, kosmetisch jedoch fast stets als angenehm empfundene, weitgehend homogene Hyperpigmentierung („Solarienbräune“) tritt fast bei allen Patienten auf. Die minimale pigmentierende Dosis beträgt bei den meisten Patienten mit atopischem Ekzem $50 \mathrm{~J} / \mathrm{cm}^{2}$ bei einmaliger Bestrahlung [15]. Zu beachten ist, dass bei einem nicht ganz kleinen Anteil der Patienten mit atopischem Ekzem dieses photoaggravierbar ist. Liegen entsprechende anamnestische Hinweise vor, sollte zur Sicherheit eine Testbestrahlung eines umschriebenen Areals mit der therapeutischen Strahlenquelle erfolgen, und zusätzlich zur MED-Bestimmung nach $24 \mathrm{~h}$, noch nach 48 oder $72 \mathrm{~h}$ Spätablesungen zum Ausschluss der Provokation einer Ekzemmorphe erfolgen. Bei Patienten mit bekannter Polymorpher Lichtdermatose (PLD) kann es zur Auslösung von Symptomen kommen, da diese Erkrankung in mehr als $2 / 3$ der Fälle durch hohe (evtl. mehrmalige) UVA1-Dosen provozierbar sind. Unter symptomatischer Therapie der PLD kann jedoch weitertherapiert werden, deren Hauterscheinungen bilden sich meist binnen weniger Tage zurück. Photosensibilisierenden Medikamente sollten vor einer UVA-1-Therapie abgesetzt werden.

Bezüglich möglicher Langzeitrisiken ist nach 10-jähriger klinischer Anwendung von UVA-1 noch nichts abschließend festzustellen. Denkbar ist eine verstärkte aktinische Elastose (Hautalterung) auch durch langwelliges UVA-1. Es konnte aber gezeigt werden, dass mittel dosiertes UVA-1 einen Einfluss auf den Kollagen-I- und -III-Metabolismus im Sinne einer Abnahme dieser Kollagene in der Haut zur Folge hat. Es kommt in vivo zur Abnahme ihrer Synthetasen bei gleichzeitiger Induktion ihrer spezifischen Proteasen. Im Gegensatz zu anderen, seit vielen Jahren in der Dermatologie routinemäßig eingesetzten Bestrahlungsverfahren (Heliotherapie, UVB, PUVA), konnte eine etwaige kanze- 
rogene Wirkung von UVA-1 am Menschen bislang nicht gezeigt werden. Tierexperimentelle Befunde an Albinomäusen zeigen immerhin, dass UVA-1 in extremen kumulativen Dosen epitheliale Tumoren auslösen kann. Auch die Rolle von UVA bei der Melanomentstehung ist Gegenstand tierexperimenteller Forschung. Kürzlich wurde über einen Einzelfall berichtet, indem es 18 Monate nach UVA-1- und anschließender Bade-PUVA-Therapie bei einem Patienten mit Urticaria pigmentosa zur Entwicklung eines malignen Melanoms kam [48]. Nach dem gegenwärtigen Kenntnisstand ist aber für UVA-1 von einem günstigen Wirkung-Nebenwirkung/Risiko-Verhältnis im Vergleich zu den klassischen UV-Therapieverfahren auszugehen [31]. Aufgund der derzeitigen Erkenntnis sollte die Indikation zur Hochdosis- und MitteldosisUVA-1-Therapie auf schwere, akut exazerbierte oder anderwärtig therapierefraktäre Fälle beschränkt bleiben, Kinder unter 6 Jahren (bei Hochdosis-Therapie Personen unter 18 Jahren) sollten nicht therapiert weden. Die Anwendung sollte auf maximal zwei Behandlungsserien von je 15 (atopisches Ekzem) bzw. 30 Bestrahlungen (Sklerodermie) pro Jahr beschränkt bleiben. Darüber hinaus sollten in der Regel Patienten mit dem Syndrom der dysplastischen Nävuszellnävi oder bekannter Melanomerkrankung sowie mit Xeroderma pigmentosum oder verwandten Erkrankungen auch von der Niedrigdosis-UVA-1-Therapie ausgeschlossen werden.

\section{Nachtrag bei Korrektur}

In einer vergleichenden Untersuchung an 22 Patienten mit Urticaria pigmentosa konnte ein gleichwertiger therapeutischer Effekt von Mitteldosis- $\left(10 \times 60 \mathrm{~J} / \mathrm{cm}^{2}\right)$ und Hochdosis- $(10 \times 130$ $\mathrm{J} / \mathrm{cm}^{2}$ ) UVA-1, der in den meisten Fällen über 6 Monate anhielt, gefunden werden. [Gobello T, Mazzanti C, Sordi D, Annessi G, Abeni D, Chinni LM, Giorlomoni GJ. Medium versus high-dose ultraviolet A1 therapy for urticaria pigmentosa: A pilot study. J Am Acad Dermatol 2003; 49:679-684]

\section{Literatur}

${ }^{1}$ Asawanonda P, Khoo LS, Fitzpatrick TB, Taylor CR. UV-A1 for keloid. Arch Dermatol 1999; 35: 348-349

${ }^{2}$ Abeck D, Schmidt T, Fesq H, Strom K, Mempel M, Brockow K, Ring J. Long-term efficacy of medium-dose UVA1 phototherapy in atopic dermatitis. J Am Acad Dermatol 2000; 42: 254-257

${ }^{3}$ von Bohlen F, Kallusky J, Woll R. Die UVA-1-Kaltlichtbehandlung der atopischen Dermatitis. Allergologie 1994; 17: 382 - 384

${ }^{4}$ Camacho NR, Sánchez JE, Martin RF, González JR, Sánchez JL. Mediumdose UVA1 phototherapy in localized scleroderma and its effect in CD34-positive dendritic cells. J Am Acad Dermatol 2001; 45: 697-699

${ }^{5}$ Dittmar HC, Pflieger F, Schöpf E, Simon JC. UVA1-Phototherapie: Pilotstudie zur Dosisfindung bei der akut exazerbierten atopischen Dermatitis. Hautarzt 2001; 52: 423-427

${ }^{6}$ Göring HD, Drabe S. Erfahrungen mit einer Mitteldosis-UVA-1-Therapie des akut exazerbierten atopischen Ekzems. Z Dermatol 1999; 85: $113-116$

${ }^{7}$ Grundmann-Kollmann M, Behrens S, Gruss C, Gottlober P, Peter RU, Kerscher ML. Chronic graft-versus-host disease refractory to immunosuppressive treatment responds to UVA1 phototherapy. J Am Acad Dermatol 2000; 42: 134-136

${ }^{8}$ Hadschiew I. UVA-1 Therapie der retikulären erythematösen Muzinose (REM). Derm Praktische Dermatologie 1998; 4: 276-278

${ }^{9}$ Hannuksela-Svahn A, Grandal OJ, Thorstensen T, Christensen OB. UVA1 for treatment of keloids. Acta Derm Venereol (Stockh.) 1999; 79: 490
${ }^{10}$ Kerscher M, Volkenandt M, Gruss C, Reuther T, von Kobyletzki G, Freitag M, Drischka T, Altmeyer P. Low-dose UVA1 phototherapy for teatment of localized scleroderma. J Am Acad Dermatol 1998; 38: 21 - 26

11 von Kobyletzki G, Drischka T, Freitag M, Hoffmann K, Altmeyer P. Ultraviolet-A1 phototherapy improves the status of the skin in cutaneous T-cell lymphoma. Br J Dermatol 1999; 140: 768 - 769

12 von Kobyletzki G, Kreuter JA, Nordmeier R, Stücker M, Altmeyer P. Treatment of idiopathic mucinosis follicularis with UVA1 coldlight. Dermatology 2000; 201: 76-77

${ }^{13}$ von Kobyletzki G, Pieck C, Hoffmann K, Freitag M, Altmeyer P. Medium-dose UVA1 cold-light phototherapy in the treatment of severe atopic dermatitis. J Am Acad Dermatol 1999; 41: 931 - 937

14 von Kobyletzki G, Uhle A, Pieck C, Hoffmann K, Altmeyer P. Acrosclerosis in patients with systemic sclerosis respond to low-dose UVA1 phototherapy. Arch Dermatol 2000; 136: 275-276

${ }^{15}$ Kowalzick L, Kleinheinz A, Weichenthal M, Neuber K, Köhler I, Grosch J, Lungwitz G, Seegeberg C, Ring J. Low dose versus medium dose UV-A1 treatment in severe atopic eczema. Acta Derm Venereol (Stockh) 1995; 75: $43-45$

${ }^{16}$ Kowalzick L, Büttner G, Neuber K, Kleinheinz A, Weichenthal M, Ring J. UVA1-Kaltlicht-Bestrahlungsbehandlung des atopischen Ekzems: Positive Dosiswirkungskorrelation. Hautnah Dermatologie 1995; 11: $374-378$

${ }^{17}$ Kowalzick L, Pönnighaus JM, Suckow M, Waldmann T. Responder und Nonresponder bei der UV-A-1-Therapie des akut exazerbierten atopischen Ekzems. Hautarzt 1997; 48: 645-647

18 Kowalzick L, Waldmann T, Suckow M, Pönnighaus JM. Dauer des Therapieerfolges bei UV-A1-Therapie des schweren atopischen Ekzems. Derm Praktische Dermatologie 1998; 4: 139-143

${ }^{19}$ Kowalzick L, Suckow M, Waldmann T, Pönnighaus JM. MitteldosisUV-A1 versus UV-B-Therapie bei Psoriasis. Zeitschr Dermatol 1999; 185: $92-94$

${ }^{20}$ Kowalzick L, Hölig O, Waldmann T, Suckow M, Ott A, Pönnighaus JM. Versagen von Mitteldosis- und Hochdosis-UV-A1 bei zwei Fällen von Urticaria pigmentosa. Akt Dermatol 1995; 25: 389-391

${ }^{21}$ Kowalzick L. UVA1 for atopic dermatitis: Medium dose superior to low dose. J Am Acad Dermatol 2001; 44: 548

${ }^{22}$ Kowalzick L, Suckow S, Mörtel J, Mischke D, Waldmann T, Pönnighaus JM. Sklerodermie en coup de Sabre: Erfolgreiche Therapie mit Calcipotriol und Medium-Dose UV-A1-Phototherapie. Akt Dermatol 2002; 28: $193-195$

${ }^{23}$ Kreuter A, Gambichler T, Avermaete A, Jansen T, Hoffman M, Altmeyer P, von Kobyletzki G, Bacharach-Buhles M. Combined treatment with calcipotriol ointment and low dose ultraviolet A1 phototherapy in childhood morphea. Pediatr Dermatol 2001; 18: 241 -245

${ }^{24}$ Kreuter JA, Gambichler T, Jansen T, Hoffmann K, Altmeyer P, von Kobyletzki G. UVA1 cold-light phototherapy of small plaque parapsoriasis. Acta Dermatovenereol (Stockh.) 2000; 80: 390-391

${ }^{25}$ Kreuter A, Jansen T, Stucker M, Herde M, Hoffmann K, Altmeyer P, von Kobyletzki G. Low-dose ultraviolet-A1 phototherapy for lichen sclerosus et atrophicus. Clin Exp Dermatol 2001; 26: 30 - 32

${ }^{26}$ Krutmann J, Czech W, Diepgen T, Niedner R, Kapp A, Schöpf E. Highdose UVA1 therapy in the treatment of patients with atopic dermatitis. J Am Acad Dermatol 1992; 26: 225 - 230

${ }^{27}$ Krutmann J, Diepgen T, Luger TA, Grabbe S, Meffert H, Sönnichsen N, Czech W, Kapp A, Stege H, Grewe M, Schöpf E. High-dose UVA1 therapy for atopic dermatitis: Results of a multicenter trial. J Am Acad Dermatol 1998; 38: 589-593

${ }^{28}$ McGrath H. UV-A1 irradiation decreases clinical disease activity and autoantibodies in patients with systemic lupus erythemathosus. Clin Exp Rheumatol 1994; 12: 129-135

${ }^{29}$ McGrath H, Martinez-Osuna P, Lee FA. Ultraviolet-A1 (340-400 nm) irradiation in systemic lupus erythematosus. Lupus 1996; 5: 269-274

${ }^{30}$ Meffert H, Sönnichsen N, Herzog M, Hutschenreuther A. UV-A1-Kaltlichttherapie des akut exazerbierten, schweren atopischen Ekzems. Dermatol Monatsschr 1992; 178: 291 - 296

${ }^{31}$ Meffert H. Stellenwert der UVA-1-Therapie. Z Hautkr 1996; 71: $721-723$

32 Molina JF, McGrath H. Longterm ultraviolet-A1 irradiation therapy in systemic lupus erthematosus. J Rheumatol 1997; 24: 1072 - 1074

${ }^{33}$ Morita A, Kobayashi K, Isomura I, Tsuji T, Krutmann J. Ultraviolet A1 $(340-400 \mathrm{~nm})$ phototherapy for scleroderma in systemic sclerosis. J Am Acad Dermatol 2000; 43: 670-674 
${ }^{34}$ Mutzhas MF, Hölzle E, Hofmann C, Plewig G. A new apparatus with high radiation energy between $320-460 \mathrm{~nm}$ : Physical description and dermatological applications. J Invest Dermatol 1981; 76: $42-47$

35 Pinton PC, Capezzera R, Zane C, DePanfilis G. Medium-dose ultraviolet A1 therapy for pityriasis lichenoides et varioliformis and pityriasis lichenoides chronica. J Am Acad Dermatol 2002; 47: 410-414

${ }^{36}$ Plettenberg H, Stege H, Megahed M, Ruzicka T, Hosokawa Y, Tsuji T, Morita A, Krutmann J. Ultraviolet A1 (340-400 nm) phototherapy for cutaneous T-cell lymphoma. J Am Acad Dermatol 1999; 41: 47-50

${ }^{37}$ Ploetz SG, Abeck D, Seitzer U, Hein R, Ring J. UVA1 for hypereosinophilic syndrome. Acta Dermatovenereol (Stockh.) 2000; 80: 221

38 Polderman MC, Huizinga TW, Le Cessie S, Pavel S. UV-A1 cold light treatment of SLE: A double blind, placebo controlled crossover trial. Ann Rheum Dis 2001; 60: 112 - 115

${ }^{39}$ Schaller M, Romiti R, Wollenberg A, Prinz B, Woerle B. Improvement of cutaneous manifestations in POEMS syndrome after UVA1 phototherapy. J Am Acad Dermatol 2001; 45: 969-970

${ }^{40}$ Schempp CM, Effinger T, Czech W, Krutmann J, Simon JC, Schöpf E. Charakterisierung von Non-Respondern bei der hochdosierten UVA1-Therapie der akut exazerbierten Atopischen Dermatitis. Hautarzt 1997; 48: 94-99

${ }^{41}$ Schmidt T, Abeck D, Boeck K, Mempel M, Ring J. UVA1 irradiation is effective in the treatment of chronic vesicular dyshidrotic hand eczema. Acta Derm Vereol (Stockh) 1998; 78: 318-319
${ }^{42}$ Sönnichsen N, Meffert H, Kunzelmann V, Audring H. UVA-1 Therapie bei subakut-kutanem Lupus erythematodes. Hautarzt 1993; 44: $723-725$

${ }^{43}$ Ständer H, Schwarz T. Ultraviolet A1 (340-400 nm) phototherapy for cutaneous T-cell lymphoma. J Am Acad Dermatol 2000; 43: 881 -

${ }^{44}$ Stege H, Schöpf E, Ruzicka T, Krutmann J. High-dose-UVA1 for urticaria pigmentosa. Lancet 1996; 347: 64

${ }^{45}$ Stege H, Berneburg M, Humke S, Klammer M, Grewe M, Grether-Beck S, Boedeker R, Diepgen T, Dierks K, Goerz G, Ruzicka T, Krutmann J. High-dose UVA1 radiation therapy for localized scleroderma. J Am Acad Dermatol 1997; 36: 938 - 944

${ }^{46}$ Stücker M, Schreiber D, Gruss C, Freitag M, von Kobyletzki G. Schwerer Verlauf einer mutilierenden pansklerotischen Sklerodermie im Kindesalter. Hautarzt 1999; 50: 131 - 135

47 Tzaneva S, Seeber A, Schwaiger M, Hönigsmann H, Tanew A. Highdose versus medium-dose UVA1 phototherapy for patients with severe generalized atopic dermatitis. J Am Acad Dermatol 2001; 45: 503-507

48 Wallenfang K, Stadler R. Assoziation zwischen UVA1 bzw. Bade-PUVA-Bestrahlung und Melanomentwicklung? Hautarzt 2001; 52 $705-707$

49 Zane C, Leali C, Airo P, de Panfilis G, Pinton PC. "High-dose" UVA1 therapy of widespread plaque-type, nodular, and erythrodermic mycosis fungoides. J Am Acad Dermatol 2001; 44: 629-633 